\title{
Identification of gene expression alterations in C. elegans adr-2 mutants using high- throughput sequencing
}

\author{
Jackson Townsend, Heather A. \\ Hundley \\ Biochemistry and Molecular Biology, Medical Sciences Program-IUSM \\ Bloomington
}

\begin{abstract}
Background and Hypothesis: RNA editing is one of several mechanisms regulating gene expression. One type of RNA editing, the deamination of adenosine to inosine, is carried out by ADAR enzymes. ADAR enzymes are essential for neural function and aberrant editing is implicated in various forms of neuropathology. C. elegans lacking the RNA editing enzyme, ADR-2, are viable allowing us to ascertain how loss of RNA editing affects neural gene expression. The effects of loss of adr-2 on neural gene expression will be analyzed in both the first larval (L1) and young adult stages. We hypothesize that the transcriptome will change depending on life stage and the presence of ADR-2.
\end{abstract}

Methods: Three replicates of neural cells isolated from wild type and adr-2(-) L1 and young adult stage animals were obtained. Total RNA was extracted from each population and mRNA was isolated using an oligo-dT bead. The mRNA was fragmented, and reverse transcribed to generate a complentary DNA (cDNA) library. The cDNA was sequenced by a facility at Indiana University. Quality of the library was evaluated using FASTqc. DE-seq2 software evaluated the differential gene expression.

Results: I examined differential gene expression in two life stages of the WT and adr-2 neural samples. After obtaining the differentially expressed genes, the portions of the transcriptome that require ADR-2 was determined. WT young adults showed increased (3715) and decreased (2504) expression of neural genes when compared to the L1 stage. Many differentially expressed genes required adr-2 ( $40 \%$ of the upregulated and $78 \%$ of the downregulated genes.) In addition, some genes were uniquely altered (631 upregulated, 196 downregulated) in the absence of adr-2.

Conclusion and Potential Impact: The life stage and presence of ADR-2 alter the neural transcriptome and this function changes throughout development. Future studies will determine whether these genes are altered due to the lack of RNA editing or binding by ADR- 2 . 